\title{
CRASHWORTHINESS OPTIMIZATION OF MULTI-CELL TRIANGULAR TUBES
}

\author{
TRAN TRONG NHAN \\ Faculty of Mechanical Engineering, Industrial University of Ho Chi Minh City; \\ trantrongnhan@iuh.edu.vn
}

\begin{abstract}
This paper aims to design the multi-cell cross-sectional thin-walled columns with two crashworthiness criteria. An explicit finite element analysis (FEA) is used to derive higher-order response surfaces for these two objectives. In the process of multiobjective crashworthiness optimization, Deb and Gupta method was utilized to find out the knee points from the Pareto solutions space. The efficiency of the crashworthiness optimization design method based on surrogate models is identified.
\end{abstract}

Keywords. Crashworthiness; Multiobjective optimization; Multi-cell; Energy absorption; Impact loading.

\section{INTRODUCTION}

Thin-walled tubes have been widely used in vehicle crashworthiness components to absorb impact energy in the past two decades. Beside square and circular tubes, several other profiles are also researched on their static or dynamic loading, such as triangular tubes [1,2,3,4], hexagonal tubes [5] and etc. The structural collapse modes of triangular tubes are different from those of square tubes. However, the forcedisplacement curve of triangular tubes are similar to those of square tubes. The crushing curves of forcedisplacement of all the profiles show that the crushing force firstly reaches an initial peak, then drops down and then fluctuates around a value of the mean crushing force. The extensional deformation has more dominant effect on the crushing responses while the quasi-inextensional mode normally occurs [6].

As a relatively new class of sectional configuration, multi-cell tubes display remarkably high capacity of energy absorption, which have latterly drawn increasing recognition in the research community and automotive industry. In this regard, Chen et al. [7] systemically studied a relative performance of single-, double- and triple-celled columns. In their study, notwithstanding the recognition of importance to wall thickness, it remains unclear what the best value is, so does the sectional width for a given constraint of mass. To increase the energy absorption, Kim [8] developed a novel type of section with various squared cells attached to the corner. An empirical objective function is constructed in terms of mean crushing force and final displacement, which allows determining their analytical derivatives regarding the size variables chosen. Such new designs show an appreciably higher crashworthiness than those more conventional designs by Chen et al. [7] Although these studies showed great promise, there is a fundamental lack of thorough design investigates on multi-celled tubes in particular when multiple crashworthiness criteria, e.g. SEA and PCF, are involved.

On the other hand, multi-objective optimization, as a more practical design methodology, aims at addressing a number of design criteria, which has become an attractive research topic in crashworthiness design recently $[9,10]$.

The present work aims at maximizing the specific energy absorption (SEA) and minimizing the peak crushing force $(\mathrm{PCF})$ for thin-walled triangular tube, where different cross-sectional tube are taken into account in an explicit finite element (FE) framework. A high-order response surface (RS) is built to exactly create the relationship between the objective functions of the SEA as well as PCF and the geometrical design variables of the sectional configurations considered. In order to obtain the optimal profiles under the crashworthiness criterion, dynamic finite element analysis code ANSYS/LS-DYNA is executed to simulate tubes and to obtain the numerical results at the design sampling points. The multiobjective optimization design is utilized to obtain the optimal configurations. 


\section{OPTIMIZATION DESIGN METHODOLOGY}

Among all the indicators of crashworthiness optimization design, the vital analytical objective was the energy-absorption. Hence, in order to estimate the energy absorption of structural unit mass $m$, Specific Energy Absorption (SEA) was formulated as:

$$
S E A=\frac{E_{A}}{m}
$$

In fact, a higher SEA indicates a better capability of energy absorption. In Equation (2), the total strain energy during crushing was estimated as

$$
E_{A}=\int_{0}^{d} P(x) d x
$$

where $P(x)$ is the instantaneous crushing force. In addition, the initial Peak Crushing Force (PCF) of multi-cell thin-walled tube was used for estimating the impact characteristics. Whereas, another crashworthiness indicator is the mean crushing force $\left(\mathrm{P}_{\mathrm{m}}\right)$ which was computed by

$$
P_{m}=\frac{E_{A}}{d}=\frac{1}{d} \int_{0}^{d} P(x) d x
$$

where $d$ is the crushing displacement at a specific time.

\subsection{Response Surface Method (RSM)}

Typical surrogate modelling technique was considered appropriate in the multivariate optimization process involving material, geometrical nonlinearities and contact-impact loading nonlinearities. The primary concept of RSM was applied to the construction of regression functions for crashworthiness indicators by using the function values at the design sampling points. The mathematical expression of RSM was expressed as

$$
y(x) \approx \tilde{y}(x)=\sum_{i=1}^{m} \beta_{i} \psi_{i}(x)
$$

where $\tilde{y}(x)$ and $y(x)$ are respectively the surrogate surface approximation and the numerical solution denoting for $\mathrm{y}(\mathrm{x}) . m$ represents the total number of basic functions $\psi_{i}(x)$, and $\beta_{i}$ is the unknown coefficient. Taking $n$ dimensional problem for example, the full linear polynomial basis function was

$$
1, x_{1}, x_{2}, \ldots, x_{n}
$$

and the full quartic polynomial basis function was expressed as

$$
\begin{aligned}
& 1, x_{1}, x_{2}, \ldots, x_{n}, x_{1}^{2}, x_{1} x_{2}, \ldots, x_{1} x_{n}, \ldots, x_{n}^{2}, x_{1}^{3}, x_{1}^{2} x_{2}, \ldots, x_{1}^{2} x_{n}, x_{1} x_{2}^{2}, \ldots, \\
& x_{1} x_{n}^{2}, \ldots, x_{n}^{3}, x_{1}^{4}, x_{1}^{3} x_{2}, \ldots, x_{1}^{3} x_{n}, x_{1}^{2} x_{2}^{2}, \ldots, x_{1}^{2} x_{n}^{2}, \ldots, x_{1} x_{2}^{3}, \ldots, x_{1} x_{n}^{3}, \ldots, x_{n}^{4}
\end{aligned}
$$

The full quartic polynomial basis function was proved to be a better choice for the regression analysis $[5,11,12,13]$. The quartic response surface models were consequently adopted in this study.

\subsection{Multi-objective optimization}

With two objectives of SEA and PCF, the multiobjective optimization problem for minimizing PCF and maximizing SEA was defined by the linear weighted average methods (LWAM) [11]. Then, the mathematical definition for the crashworthiness optimization in terms of the LWAM was given as

$$
\begin{cases}\text { Minimize } & F(t, a)=w \frac{P C F(t, a)}{P C F^{*}}+(1-w) \frac{S E A^{*}}{S E A(t, a)} \\ \text { s.t } & \mathrm{w} \in[0,1] \\ & 1 \leq \mathrm{t} \leq 2.2 \mathrm{~mm} \\ & 80 \leq \mathrm{a} \leq 100 \mathrm{~mm}\end{cases}
$$

where $S E A^{*}$ and $P C F^{*}$ are the given normalizing values for each cross-sectional profile. 


\subsection{Knee point}

In some certain cases, the designer must choose the most preferred solution (termed as "knee point") from optimal solutions to meet their requirement. Several methods were proposed to determine a "knee point" from Pareto set such as Turevsky and Suresh [14] and Sun et al. [15]. However, if there is a great deviation among the orders of magnitude of different objectives, these method [15] seems to be less effective. A modified multi-objective evolutionary algorithm suggested by Branke et al. [16] was utilized to seek the knee regions. Deb and Gupta [17] have recently suggested a solution to find a "knee point" with maximum bend-angle, which was mathematically given as,

$$
\text { Maximize } \theta\left(x, x_{L}, x_{R}\right)=\theta_{L}-\theta_{R}
$$

where $\theta_{L}=\arctan \frac{f_{2}\left(x_{L}\right)-f_{2}(x)}{f_{1}(x)-f_{1}\left(x_{L}\right)}$ and $\theta_{R}=\arctan \frac{f_{2}(x)-f_{2}\left(x_{R}\right)}{f_{1}\left(x_{R}\right)-f_{1}(x)}$ are the left and right bend-angle of $x$.

\section{NUMERICAL SIMULATION AND CRASHWORTHINESS OPTIMIZATION}

\subsection{Numeric simulation}

In this section, FE model was carried out by ANSYS/LS-DYNA to simulate the triangular multi-cell thin-walled tubes subjected to axial dynamic loading with 4, 6 and 9 cells (as shown in Fig. 1). The sidelength $a$ of the cross-sections and the thickness $t$ were chosen to be design variables, and the design interval was given in Equation (7). The total length $L_{0}$ of all the tubes is $250 \mathrm{~mm}$.

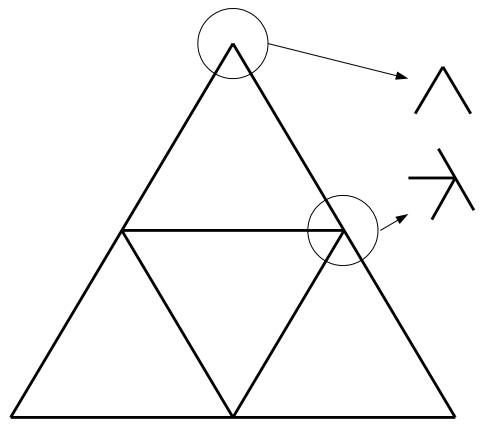

(a): Tube type I

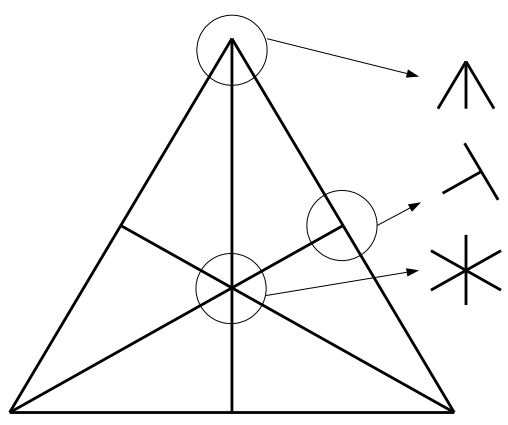

(b): Tube type II

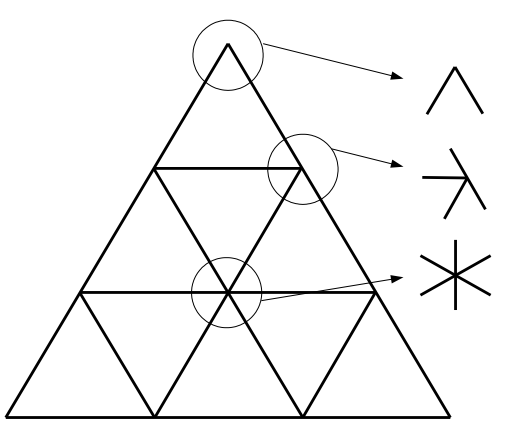

(c): Tube type III

Fig. 1. Cross-sectional geometry of triangular multi-cell tube and typical angle element.

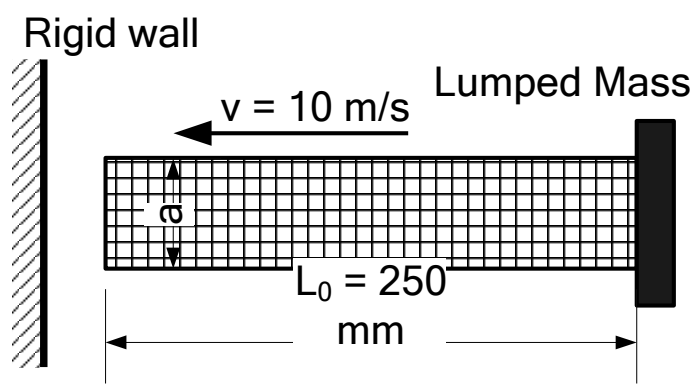

Fig. 2. Schematic of the computational model. 

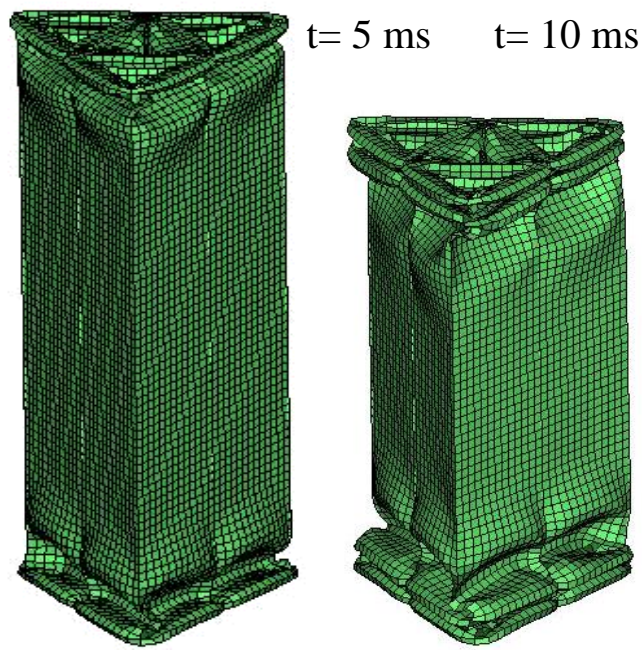

$$
\mathrm{t}=15 \mathrm{~ms}
$$

$\mathrm{t}=20 \mathrm{~ms}$
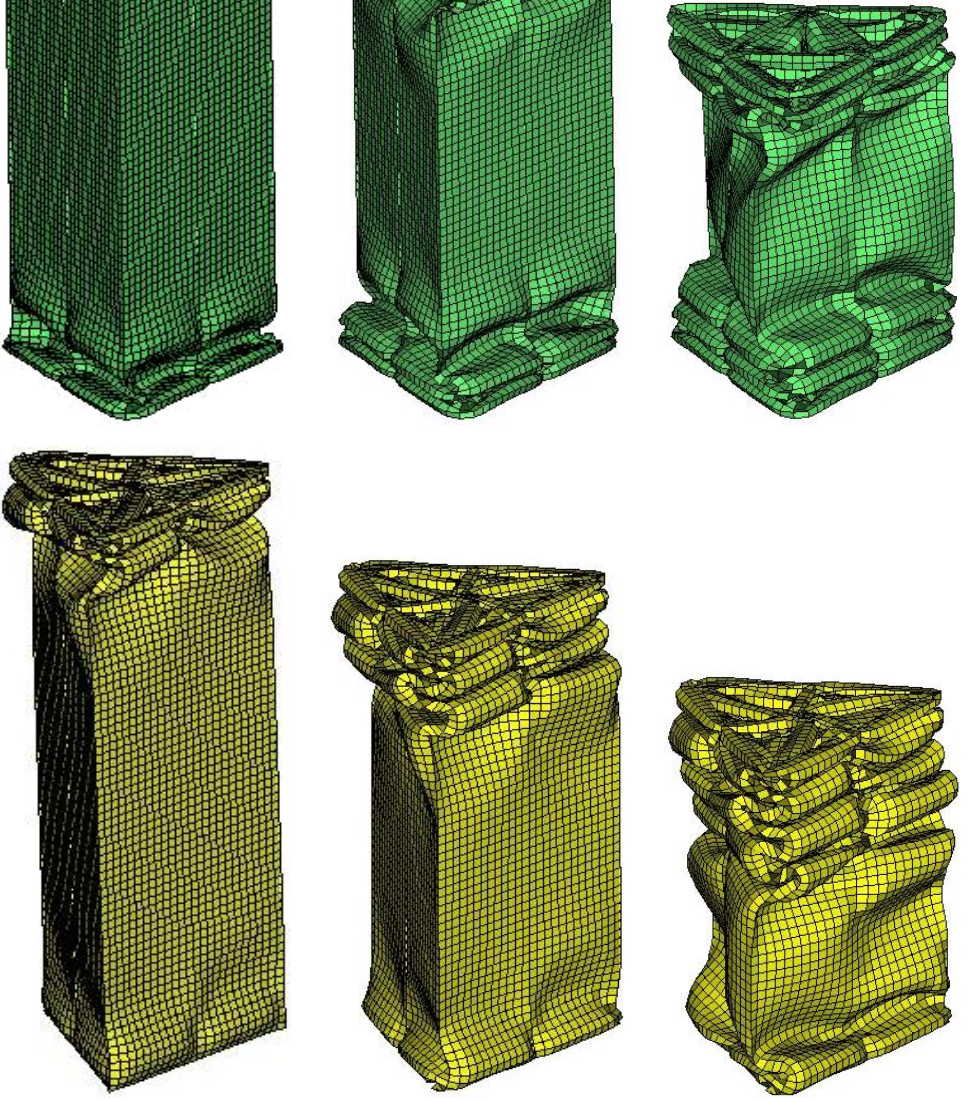

Tube I
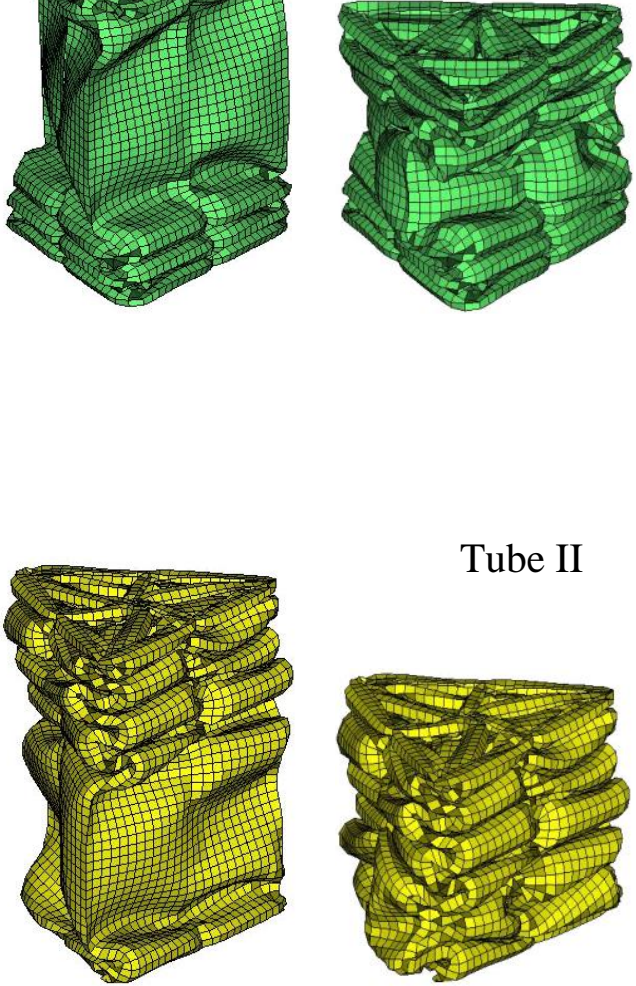

Tube II
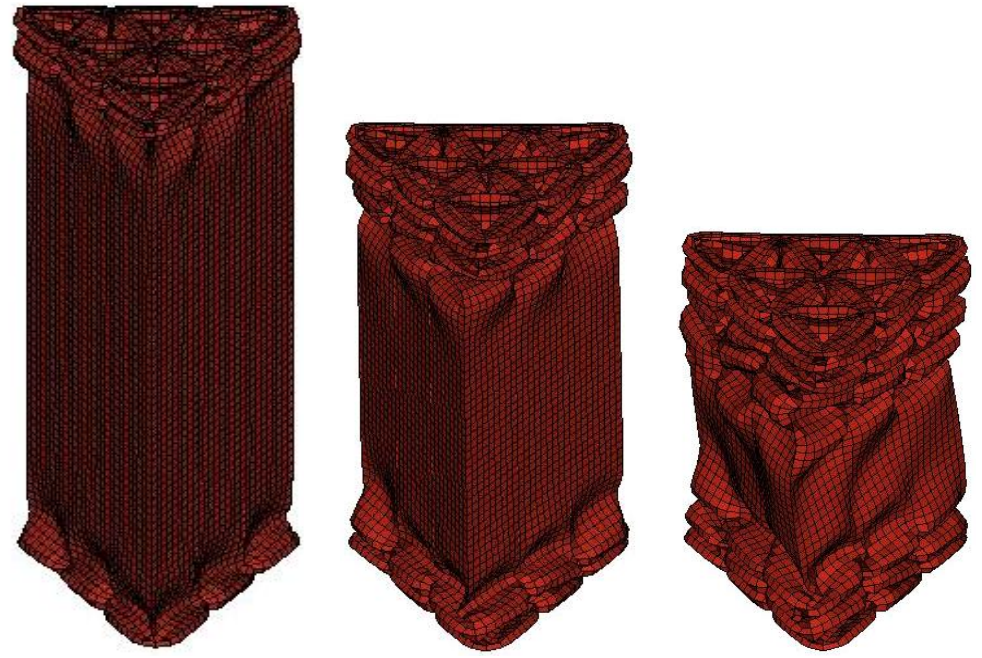

Tube III

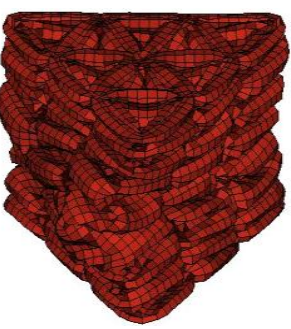

Fig. 3. Deformation process of three tubes. 

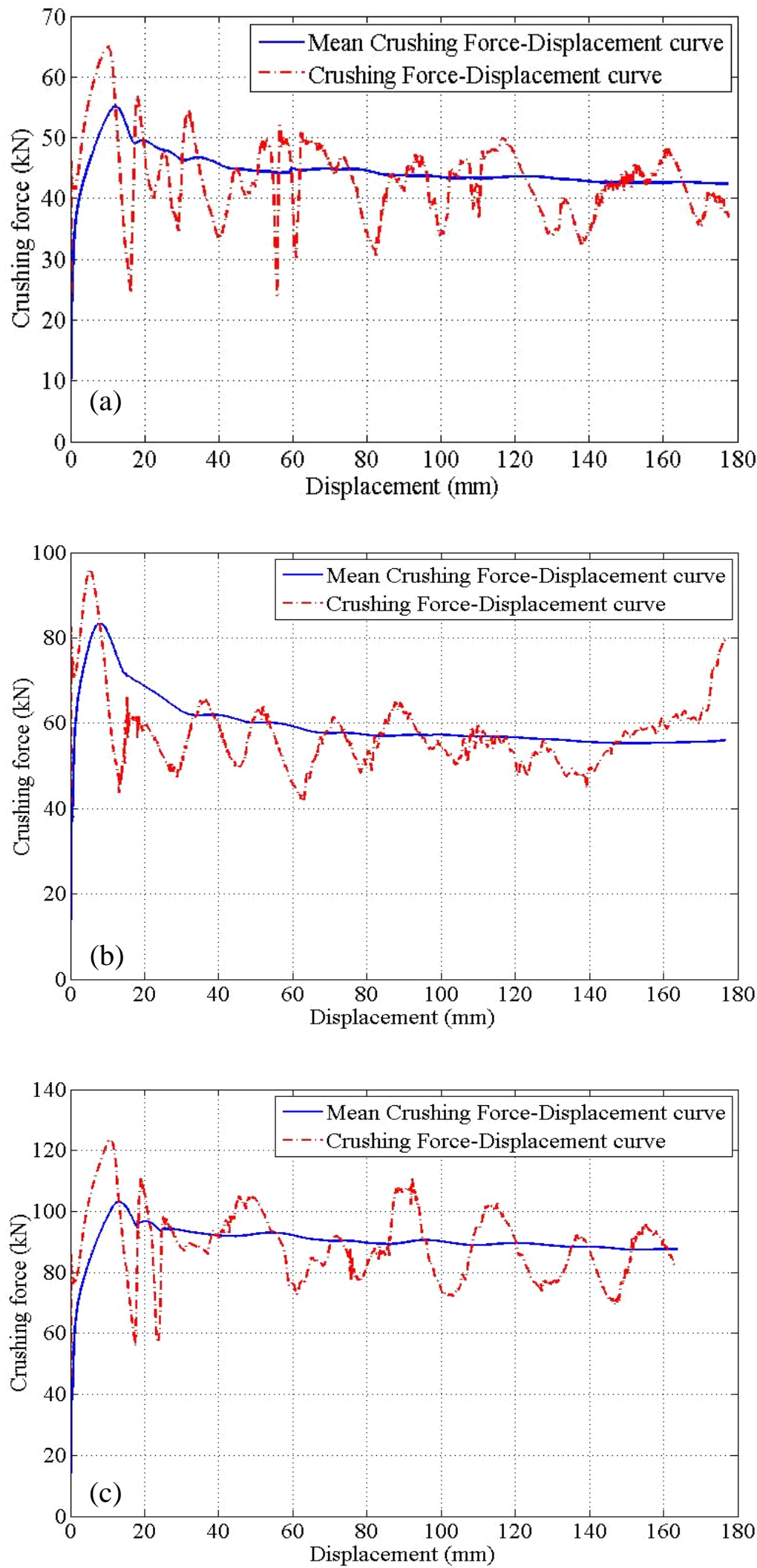

Fig. 4. The crushing force-displacement curve of (a) tube I, (b) tube II and (c) tube III. 
In this study, the thin-walled tubes were modelled with the Belytschko-Tsay four-node shell element with the optimal mesh density of $2.5 \times 2.5 \mathrm{~mm}$. The material AA6060 T4 was modelled with material model \#24 (Mat_Piecewise_Linear_Plasticity) with mechanical properties: Young's modulus $E=$ $68200 \mathrm{MPa}$, initial yield stress $\sigma_{y}=80 \mathrm{MPa}$, ultimate stress $\sigma_{u}=173 \mathrm{MPa}$, Poisson's ration $v=0.3$, and power law exponent $n=0.23$ [18]. Since the aluminium was insensitive to the strain rate effect, this effect was neglected in the finite element analysis. An automatic node to surface contact between thin-walled tube and rigid wall was defined to simulate the real contact. Alternatively, an automatic single surface contact algorithm was utilized for the self contact among the shell elements to avoid interpenetration of folding generated during the axial collapse. In the contact definition, a friction coefficient of 0.3 among all surfaces was employed. To generate enough kinetic energy, one end of tube was attached with a lumped mass of $500 \mathrm{~kg}$ whereas another end impacted onto a rigid wall with an initial velocity of $10 \mathrm{~m} / \mathrm{s}$. The schematic of the computational model was shown in Fig. 2.

All of the tubes were axial symmetric structures. Despite the same length, same side-length and same thickness, the three tubes were different in weight. Tube I is the lightest one while tube III is the heaviest. The axial crushing of multi-cell tubes was presented with a displacement equal to about $70 \%$ of the initial length. Fig. 3 shows the deformation process of three tubes at different time. Sometimes the exact value of the effective crushing distance on the crushing force-displacement curve was not unique. The corresponding crushing force-displacement curves of three tubes were also shown in Fig. 4. After reaching the initial peak and before rising steeply whenever the deformation capacity is exhausted at the effective crushing distance, the crushing force fell sharply and then fluctuated periodically and around the values of the mean crushing force in correspondence with the formation; and finally completed the collapse of folds one by one.

\subsection{Crashworthiness optimization}

For obtaining the response functions of SEA and PCF, a series of 25 design sampling points (based on $a$ and $t$ ) were selected in the design space to provide sampling design values for FEA and regression analysis of three types of tubes (Table 1) so as to obtain the response surface of the SEA and PCF. Fig. 5 shows that the SEA's and PCF's RS of tube type I, II and III cases behave monotonically over the design domain. In addition, the curves in Fig 6 illustrate the variation of SEA and $P_{m}$ with changes in weight. Meanwhile, energy absorptions of the tube type I and III were better than that of tube type II.

The Pareto sets for these three cross-sectional profiles was obtained by changing the weight coefficient $w$ in Eq. (7), and the Pareto frontiers were plotted in Fig. 7. In fact, any point on the Pareto frontier can be an optimum. As a result, some methods were proposed to determine the best solution (knee point) which has a large trade-off value in comparison with other Pareto-optimal points. In this case, methods of $[16,17]$ were utilized to determine the knee region and the knee point, respectively. The results of expression (8) showed that Pareto solutions (Knee points) for tube type I, II and III were 0.7924, 0.7818 and 0.7773 , respectively. The detailed design parameters for this optimal design and its corresponding FEA results are summarized in Table 2. We can find that the errors between FEA results and the metamodels are less than $1 \%$. This indicates that the quartic polynomial functions for multi-cell triangular tubes are accurate. The error of SEA is bigger than that of PCF for the optimal design of these tubes. We can easily find that the errors of SEA and PCF of the optimal design of tube type III are bigger than that of tube type I, respectively

Therefore, the FE simulation value and RS approximate value at the Knee points were exactly close to each other. According to the relationship among the weighted average method and those of [16,17], these optimal results were plotted in Fig. 7. 
Table 1. Design matrix of three types of tube for crashworthiness.

\begin{tabular}{|c|c|c|c|c|c|c|c|c|}
\hline \multirow[b]{2}{*}{$\mathrm{n}$} & \multirow[b]{2}{*}{$\mathrm{t}(\mathrm{mm})$} & \multirow[b]{2}{*}{$\begin{array}{c}\mathrm{a} \\
(\mathrm{mm})\end{array}$} & \multicolumn{2}{|c|}{ Tube type I } & \multicolumn{2}{|c|}{ Tube type II } & \multicolumn{2}{|c|}{ Tube type III } \\
\hline & & & $\begin{array}{c}\text { SEA } \\
(\mathrm{kJ} / \mathrm{kg})\end{array}$ & $\begin{array}{l}\text { PCF } \\
(\mathrm{kN})\end{array}$ & $\begin{array}{c}\mathrm{SEA} \\
(\mathrm{kJ} / \mathrm{kg})\end{array}$ & $\begin{array}{l}\text { PCF } \\
(\mathrm{kN})\end{array}$ & $\begin{array}{c}\text { SEA } \\
(\mathrm{kJ} / \mathrm{kg})\end{array}$ & $\begin{array}{l}\text { PCF } \\
(\mathrm{kN})\end{array}$ \\
\hline 1 & 1 & 80 & 18.262 & 39.362 & 17.356 & 49.572 & 23.339 & 52.592 \\
\hline 2 & 1.3 & 80 & 20.226 & 52.274 & 19.876 & 65.965 & 25.227 & 69.599 \\
\hline 3 & 1.6 & 80 & 22.774 & 65.287 & 21.86 & 82.467 & 28.199 & 85.801 \\
\hline 4 & 1.9 & 80 & 23.854 & 77.82 & 22.003 & 98.125 & 29.092 & 101.135 \\
\hline 5 & 2.2 & 80 & 24.732 & 88.936 & 23.107 & 111.611 & 29.644 & 114.541 \\
\hline 6 & 1 & 85 & 17.635 & 42.151 & 16.842 & 53.133 & 22.753 & 56.038 \\
\hline 7 & 1.3 & 85 & 19.491 & 55.941 & 19.272 & 70.384 & 24.273 & 74.202 \\
\hline 8 & 1.6 & 85 & 21.079 & 69.78 & 20.924 & 88.192 & 26.388 & 92.462 \\
\hline 9 & 1.9 & 85 & 22.231 & 82.904 & 21.602 & 104.633 & 27.564 & 109.979 \\
\hline 10 & 2.2 & 85 & 23.891 & 94.376 & 22.693 & 118.899 & 28.107 & 125.572 \\
\hline 11 & 1 & 90 & 16.689 & 48.183 & 16.559 & 60.979 & 21.338 & 64.544 \\
\hline 12 & 1.3 & 90 & 18.63 & 63.406 & 18.9 & 69.095 & 24.05 & 85.478 \\
\hline 13 & 1.6 & 90 & 19.883 & 80.385 & 20.273 & 99.84 & 25.536 & 107.641 \\
\hline 14 & 1.9 & 90 & 21.425 & 97.058 & 20.679 & 120.552 & 26.272 & 128.512 \\
\hline 15 & 2.2 & 90 & 22.916 & 111.899 & 21.524 & 138.568 & 26.42 & 146.48 \\
\hline 16 & 1 & 95 & 16.092 & 50.984 & 15.253 & 65.978 & 20.932 & 68.196 \\
\hline 17 & 1.3 & 95 & 17.793 & 67.207 & 16.954 & 83.194 & 22.371 & 90.665 \\
\hline 18 & 1.6 & 95 & 19.279 & 85.237 & 18.229 & 105.95 & 24.579 & 114.112 \\
\hline 19 & 1.9 & 95 & 20.865 & 102.754 & 19.146 & 128.907 & 25.306 & 135.856 \\
\hline 20 & 2.2 & 95 & 22.276 & 117.81 & 19.71 & 148.295 & 25.948 & 153.825 \\
\hline 21 & 1 & 100 & 15.569 & 50.155 & 14.398 & 63.148 & 19.337 & 66.649 \\
\hline 22 & 1.3 & 100 & 17.083 & 66.443 & 16.139 & 83.857 & 20.011 & 87.852 \\
\hline 23 & 1.6 & 100 & 19.26 & 83.029 & 17.748 & 104.85 & 22.979 & 110.016 \\
\hline 24 & 1.9 & 100 & 20.628 & 98.954 & 18.55 & 124.628 & 23.92 & 130.951 \\
\hline 25 & 2.2 & 100 & 21.053 & 113.035 & 18.715 & 142.093 & 24.152 & 157.552 \\
\hline
\end{tabular}

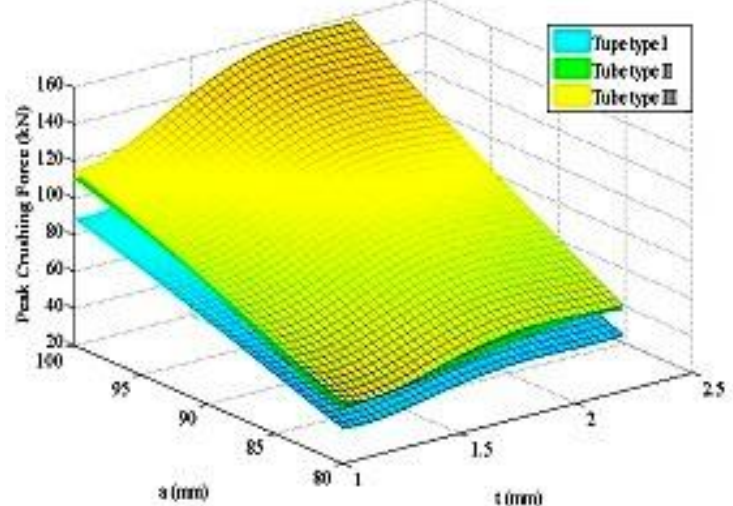

(a)

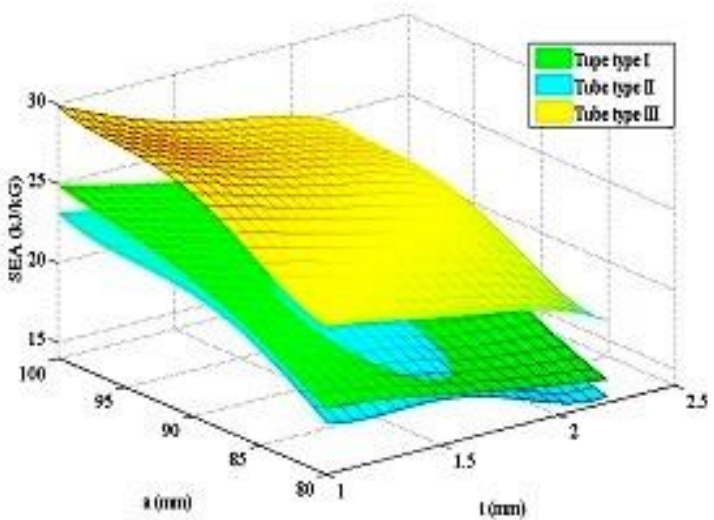

(b)

Fig. 5. The response surface of (a) Peak crushing force; (b) SEA. 


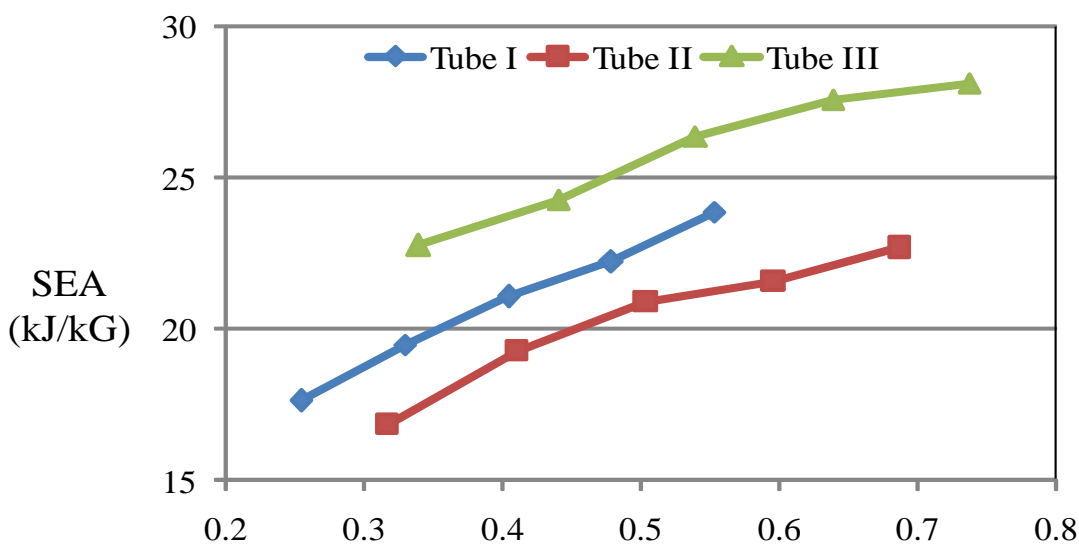

(a)

Structural Weight $(\mathrm{kG})$

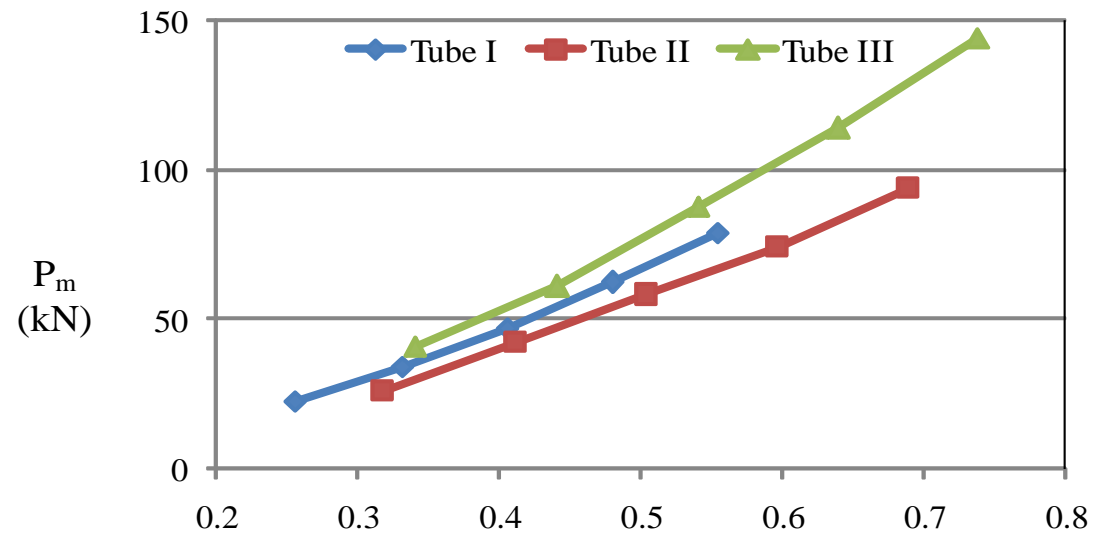

(b)

Structural Weight $(\mathrm{kG})$

Fig. 6. (a) SEA vs Structural weight, (b) Pm vs Structural weight.

Table 2. Optimal results by using method of Deb and Gupta (Knee point).

\begin{tabular}{ccccc}
\hline $\begin{array}{c}\text { Type of } \\
\text { cross-section }\end{array}$ & Terms & $\begin{array}{c}\text { Optimal design } \\
\text { variables }(\mathrm{mm})\end{array}$ & $\begin{array}{c}\text { SEA } \\
(\mathrm{kJ} / \mathrm{kN})\end{array}$ & $\begin{array}{c}\text { PCF } \\
(\mathrm{kN})\end{array}$ \\
\hline \multirow{3}{*}{ Type I } & Approximate value & & 19.897 & 49.315 \\
& FE analysis value & $\mathrm{t}=1.23, \mathrm{a}=80$ & 19.715 & 49.088 \\
& RE & & 0.766 & 0.462 \\
Type II & Approximate value & & 25.782 & 63.436 \\
& FE analysis value & $\mathrm{t}=1.25, \mathrm{a}=80$ & 25.217 & 63.056 \\
& RE & & 0.691 & 0.603 \\
Type III & Approximate value & & 25.100 & 63.267 \\
& FE analysis value & $\mathrm{t}=1.21, \mathrm{a}=80$ & 24.800 & 64.767 \\
& RE & & 0.780 & 0.467 \\
\hline
\end{tabular}



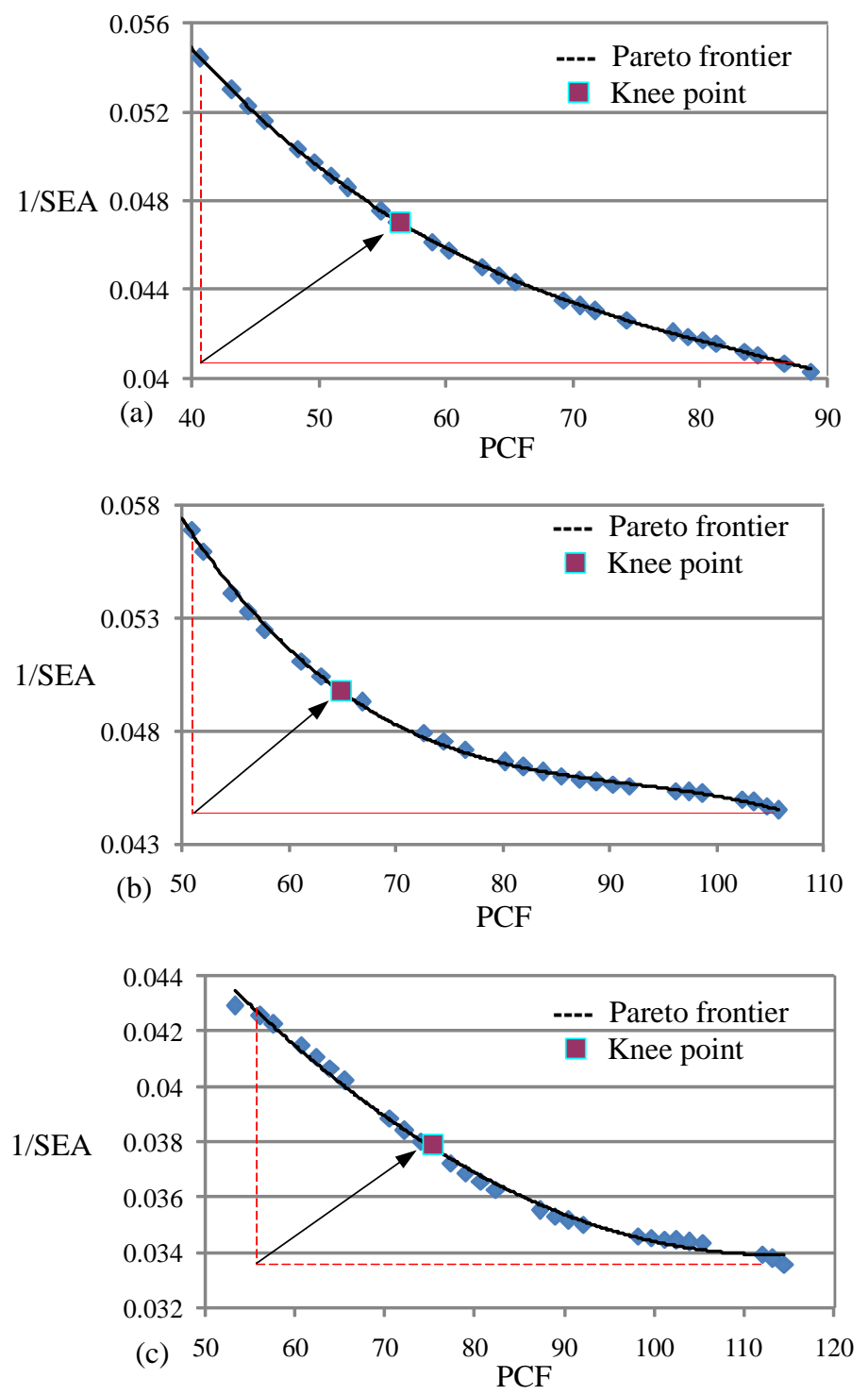

Fig. 7. Pareto spaces for multi-objective optimization: (a) tube type I; (b) tube type II and (c) tube type III.

From Fig. 7, it can be seen the Pareto fronts are convex, which simply indicate that the use of typical linear weighted method is appropriate. Besides, the method of Deb and Gupta proves fairly effective to search for a best solution from Pareto front curve.

\section{CONCLUSIONS}

In this study, the multi-cell triangular tubes with three different cross-sectional configurations have been investigated under axial crushing loads by using the nonlinear finite element code LS-DYNA. Numerical results showed that tube types I and III were better than tube type II in the aspect of energy absorption. Simultaneously, the stable and progressive folding deformation patterns appeared for all the three types of tubes.

The two RS models of PCF and SEA for each tube were constructed. Pareto sets were obtained by using the linear weighted average methods (LWAM). In this paper, the Pareto solutions of three types of tubes were identified to seek out the knee points. The relative errors between RS approximate value and $\mathrm{FE}$ analysis value at the Knee points were obtained and those were also acceptable. The result of this work showa the efficiency of the crashworthiness optimization design method based on the surrogate models and the numerical analysis techniques. 


\section{REFERENCES}

[1] M. Krolak, K. Kowal-Michalska, R. Mania, J. Swiniarski, Experimental tests of stability and load carrying capacity of compressed thin-walled multi-cell columns of triangular cross-section, Thin-Walled Structures 45 (2007) 883-887.

[2] Y. Liu, Collapse behaviour and simplified modeling of triangular crosssection columns, Indian Journal of Engineering and Materials Sciences 16 (2009) 71-78.

[3] W. Hong, F. Jin, J. Zhou, Z. Xia, Y. Xu, L. Yang, Q. Zheng, H. Fan, Quasi-static axial compression of triangular steel tubes, Thin-Walled Structures 62 (2013) 10-17.

[4] W. Hong, H. Fan, Z. Xia, F. Jin, Q. Zhou, D. Fang, Axial crushing behaviors of multi-cell tubes with triangular lattices, International Journal of Impact Engineering 63 (2014) 106-117.

[5] S. Hou, Q. Li, S. Long, X. Yang, W. Li, Design optimization of regular hexagonal thin-walled columns with crashworthiness criteria, Finite Elements in Analysis and Design 43 (2007) 555-565.

[6] W. Abramowicz, Thin-walled structures as impact energy absorbers, Thin-Walled Structures 41 (2003) 91-107.

[7] W. Chen, T. Wierzbicki, Relative merits of single-cell, multi-cell and foam-filled thin-walled structures in energy absorption, Thin-Walled Structures 39 (2001) 287-306.

[8] H.-S. Kim, New extruded multi-cell aluminum profile for maximum crash energy absorption and weight efficiency, Thin-Walled Structures 40 (2002) 311-327.

[9] H.R. Zarei, M. Kröger, Multiobjective crashworthiness optimization of circular aluminum tubes, Thin-Walled Structures 44 (2006) 301-308.

[10] X. Liao, Q. Li, X. Yang, W. Zhang, W. Li, Multiobjective optimization for crash safety design of vehicles using stepwise regression model, Structural and Multidisciplinary Optimization 35 (2008) 561-569.

[11] S. Hou, Q. Li, S. Long, X. Yang, W. Li, Multiobjective optimization of multi-cell sections for the crashworthiness design, International Journal of Impact Engineering 35 (2008) 1355-1367.

[12] S. Hou, Q. Li, S. Long, X. Yang, W. Li, Crashworthiness design for foam filled thin-wall structures, Materials \& Design 30 (2009) 2024-2032.

[13] S. Hou, X. Han, G. Sun, S. Long, W. Li, X. Yang, Q. Li, Multiobjective optimization for tapered circular tubes, Thin-Walled Structures 49 (2011) 855-863.

[14] I. Turevsky, K. Suresh, Efficient generation of pareto-optimal topologies for compliance optimization, International Journal for Numerical Methods in Engineering 87 (2011) 1207-1228.

[15] G. Sun, G. Li, S. Zhou, H. Li, S. Hou, Q. Li, Crashworthiness design of vehicle by using multiobjective robust optimization, Structural and Multidisciplinary Optimization 44 (2011) 99-110.

[16] J. Branke, K. Deb, H. Dierolf, M. Osswald, Finding Knees in Multi-objective Optimization, in: X. Yao, E. Burke, J. Lozano, J. Smith, J. Merelo-Guervós, J. Bullinaria, J. Rowe, P. Tiňo, A. Kabán, H.-P. Schwefel (Eds.), Parallel Problem Solving from Nature - PPSN VIII, Springer Berlin Heidelberg, 2004, pp. 722-731.

[17] K. Deb, S. Gupta, Understanding knee points in bicriteria problems and their implications as preferred solution principles, Engineering Optimization 43 (2011) 1175-1204.

[18] S.P. Santosa, T. Wierzbicki, A.G. Hanssen, M. Langseth, Experimental and numerical studies of foam-filled sections, International Journal of Impact Engineering 24 (2000) 509-534.

Received on January 23 - 2017

Revised on July $18-2017$ 